\title{
Increased reporting of fatal pneumonitis associated with immune checkpoint inhibitors: a WHO pharmacovigilance database analysis
}

\author{
To the Editor:
}

The discovery of immune checkpoint inhibitors (ICIs), which include anti-programmed cell death protein-1 (PD-1), its ligand (PD-L1) and anti-cytotoxic T cell lymphocyte-associated protein-4 (CTLA-4), has transformed the field of oncology, with indications continuing to increase. For example, anti-PD1 and anti-CTLA-4 ICIs, which include nivolumab, pembrolizumab and ipilimumab, are US Food and Drug Administration/European Medicines Agency approved for a variety of cancers, such as melanoma and non-small cell lung carcinoma (NSCLC) [1]. Considered the Achilles' heel of ICIs, however, are immune-related adverse events (irAEs) (10-60\% high grade) that represent an inflammatory response that can affect multiple organ systems, which can be fatal $(0.3-1.3 \%)[2]$.

Among these irAEs is ICI-related pneumonitis, which is a serious adverse event with reports of up to $1 \%$ to $4 \%$ of patients on ICI monotherapy and $4 \%$ to $7 \%$ on combination therapy $[3,4]$. Most of the available data were derived from clinical trials that may not be representative of a real-world population and settings. As such, reports of ICI-pneumonitis in the international pharmacovigilance database, Vigibase, were retrospectively reviewed to characterise their main features and factors associated with death.

Our study is a descriptive analysis based on adverse drug reactions (ADR) reported within VigiBase, the World Health Organization global deduplicated individual case safety reports (ICSRs) database, originating from more than 130 countries [5-7]. VigiBase contains over 18 million ICSRs submitted by national pharmacovigilance centres since 1967. These reports mainly originate from real-life populations with different sources, including healthcare professionals, patients and pharmaceutical companies. The use of confidential electronically processed patient data was approved by the French National Commission for Data Protection and Liberties (reference: 1922081). This study is part of NCT03492242 [5-7].

We performed a search for ADRs associated with anti-CTLA-4, anti-PD1, anti-PD-L1, and their combinations. We searched all ICI-pneumonitis according to the Medical Dictionary for Drug Regulatory Activities (MedDRA) using the preferred term "pneumonitis", up to 4 November, 2018. ICI-pneumonitis specifically considered in the analysis were those reported as "suspected" secondary to ICI. Demographic and clinical data were collected and reviewed. Characteristics of cases were described in terms of mean $\pm \mathrm{SD}$ or medians (with interquartile ranges (IQR)) for quantitative variables, and in terms of effective and proportion for qualitative ones. To compare fatal versus non-fatal pneumonitis, unpaired $\mathrm{t}$-test, Mann-Whitney and $\chi^{2}$-tests were calculated, as appropriate ( $R$-software).

Between inception through November 04, 2018, a total of 1694 ICI-pneumonitis were identified with a marked increase in reporting of ICI-pneumonitis of approximately 4 cases per month between 2010 and 2014 to 71 cases per month in 2018. The majority of cases originated from North America (47.5\%) and Europe $(39.8 \%)$ and were reported predominantly by healthcare providers $(n=1277 / 1545,82.7 \%)$ in a non-clinical trial setting $(\mathrm{n}=1596 / 1619,98.6 \%)$. Patients with ICI-pneumonitis were mostly men ( $\mathrm{n}=946 /$ $1694,63.6 \%$ ) with a median age of 65 years (range $7-103 ; n=1130 / 1694)$. Patients mainly received ICI for lung cancer $(55.9 \%, n=776 / 1388)$ and melanoma $(22.1 \%, n=307 / 1388)$. There were $88.1 \%(n=1492 / 1694)$

@ERSpublications

There was an increased reporting of immune checkpoint inhibitor (ICI)-pneumonitis over the past few years with earlier time to onset of fatal ICI-pneumonitis, which was associated with more respiratory failure and tumour progression http://bit.ly/32GW51a

Cite this article as: Moey MYY, Gougis P, Goldschmidt V, et al. Increased reporting of fatal pneumonitis associated with immune checkpoint inhibitors: a WHO pharmacovigilance database analysis. Eur Respir J 2020; 55: 2000038 [https://doi.org/10.1183/13993003.00038-2020]. 
of patients who received ICI monotherapy (71.8\% anti-PD1, 10.6\% anti-PD-L1 and 5.7\% anti-CTLA-4) and $11.9 \%(n=202 / 1694)$ combination therapy (mainly anti-CTLA-4 and anti-PD1). ICI were the only suspected drug inducing pneumonitis in $87.7 \%(\mathrm{n}=1486 / 1694)$ of the cases. Time to onset (TTO) was available in $336 / 1694(19.8 \%)$ patients. In these patients, median TTO of ICI-pneumonitis was 43 days (range 0-3345). Concurrent irAEs occurred in 390/1694 (23.0\%) patients, mainly represented by gastrointestinal $(29.2 \%, \mathrm{n}=114 / 390)$ and endocrine $(24.9 \%, \mathrm{n}=97 / 390)$ irAEs.

In our analysis, serious adverse events, defined by life threatening, prolonged hospitalisation or physical disability were observed in all cases, leading to death in 297/1694 (17.5\%) of ICI-pneumonitis cases. There were no significant differences in gender, age, country of reporting, reporter type (healthcare, non-healthcare professional), setting (clinical, non-clinical trial setting) and year of reporting (starting 2011 and ending 2018) for fatal versus non-fatal ICI-related pneumonitis cases (table 1).

There were no preferential seasons for the onset of fatal ICI-pneumonitis $(p=0.54)$. There was increased report in ICI-pneumonitis deaths with an average of 10 cases per month in 2017-2018 versus 1 case per month between 2010 and 2014. Use of ICI combination versus ICI monotherapy and type of monotherapy (anti-PD1, anti-PD-L1 and anti-CTLA4) were similar in fatal versus non ICI-pneumonitis cases (table 1), but fatality was increased in patients with lung cancer $(60.3 \%, 158 / 262$ versus $54.9 \%, 618 / 1126)$ compared to melanoma $(14.9 \%, 39 / 262$ versus $23.8 \%, 268 / 1126$; $\mathrm{p}=0.0003)$. Within anti-PD1 monotherapies, fatality was not different per ICI dosage $(\mathrm{p}=0.58)$; however, it was higher among pembrolizumab versus nivolumab treated patients $(22.4 \%, 92 / 411$ versus $15.7 \%, 126 / 805$, respectively; $\mathrm{p}=0.004 ; \mathrm{n}=54 / 185$ versus $17.5 \%, \mathrm{n}=80 / 456 ; \mathrm{p}=0.001$ in lung cancer subset).

TTO was statistically different between fatal and non-fatal cases with fatal ICI- pneumonitis cases occurring with a shorter TTO than non-fatal cases (median 24 days (IQR 12-41 days) versus 53 days (IQR 21-132.75), respectively; $\mathrm{p}<0.0001)$. To further categorise ICI-pneumonitis, we determined concomitant conditions. Concomitant irAEs were identified in $26.2 \%(n=78 / 297)$ of fatal ICI-pneumonitis ICSRs and included colitis $(8.1 \%, \mathrm{n}=24 / 297)$ and dermatitis $(6.7 \%, \mathrm{n}=20 / 297)$ among others. There was $29.8 \%$ $(\mathrm{n}=505 / 1694)$ of all ICI-pneumonitis cases with other conditions (table 1). Concomitant conditions were associated with $52.9 \%(\mathrm{n}=157 / 297)$ of fatal ICI-pneumonitis, which were sepsis-related in $26.8 \%(\mathrm{n}=42 /$ 157) of cases. Fatal cases were associated with more respiratory failure, thromboembolism and tumour progression (table 1).

To our knowledge, this study represents the largest international description and characterisation of ICI-pneumonitis cases to date. We observed a significant increase of ICI-pneumonitis with unchanged fatality rate over years. We observed an earlier TTO for fatal ICI-pneumonitis versus non-fatal cases which emphasises the need for understanding the biological mechanisms, identifying any risk factors and temporalities that can aid in the diagnosis and effective management in these patients.

A meta-analysis comparing ICI versus conventional chemotherapy in randomised controlled trials $(\mathrm{n}=7246$ patients) found a significantly higher risk of high-grade and all-grade ICI-pneumonitis (OR 4.39, $\mathrm{p}=0.003$ and OR 2.46, $\mathrm{p}=0.007$, respectively) in the ICI treatment arm [8]. In comparison to dermatological or endocrine-irAEs which may be linked to better prognosis [9], in lung cancer, ICI-pneumonitis accounted for $35 \%$ of anti-PD1 and anti-PD-L1-related deaths [10-12]. In our study, we observed a higher incidence of ICI-pneumonitis which is suspected due to a combination of increase use of ICI and awareness of irAEs. Fatal ICI-pneumonitis cases were also seen in lung cancer patients, which has been suggested to be a result of multiple factors including effect of radiation synergy and a pulmonary tumour burden affecting the microenvironment and inflammatory response $[3,4,13]$.

Similar to previous literature, there is a wide range of TTO to ICI-related pneumonitis which may be affected by common overlapping symptoms and radiographic features that may mimic underlying pulmonary conditions such as concomitant obstructive or restrictive lung disease, pulmonary infections but also lung cancer progression/hyperprogression, and a lack of consensus terminology to specifically describe and define ICI-related pneumonitis [3, 4, 14]. Early diagnosis aided by radiographic evidence would be pivotal for immediate management since fatality from ICI-related pneumonitis can occur earlier in the treatment course as observed in our study. For example, obtaining chest computed tomography (CT) prior to the second ICI administration could be of utility in order to monitor for the presence or development of specific pulmonary radiographic findings, such as ground-glass opacities/patchy consolidations, before the onset of respiratory symptoms and/or failure. Chest CT surveillance should also be strongly considered, especially in patients treated for lung cancer receiving pembrolizumab and/or with respiratory comorbidities to identify concomitant infection and early cancer hyperprogression [15].

Although this is the largest description of ICI-related pneumonitis to date, there are limitations to the study. First, the absolute incidence of ICI-related pneumonitis cannot be assessed because VigiBase does 
TABLE 1 Main characteristics of fatal versus non-fatal pneumonitis associated with immune checkpoint inhibitors (ICls) in VigiBase through November 04, 2018

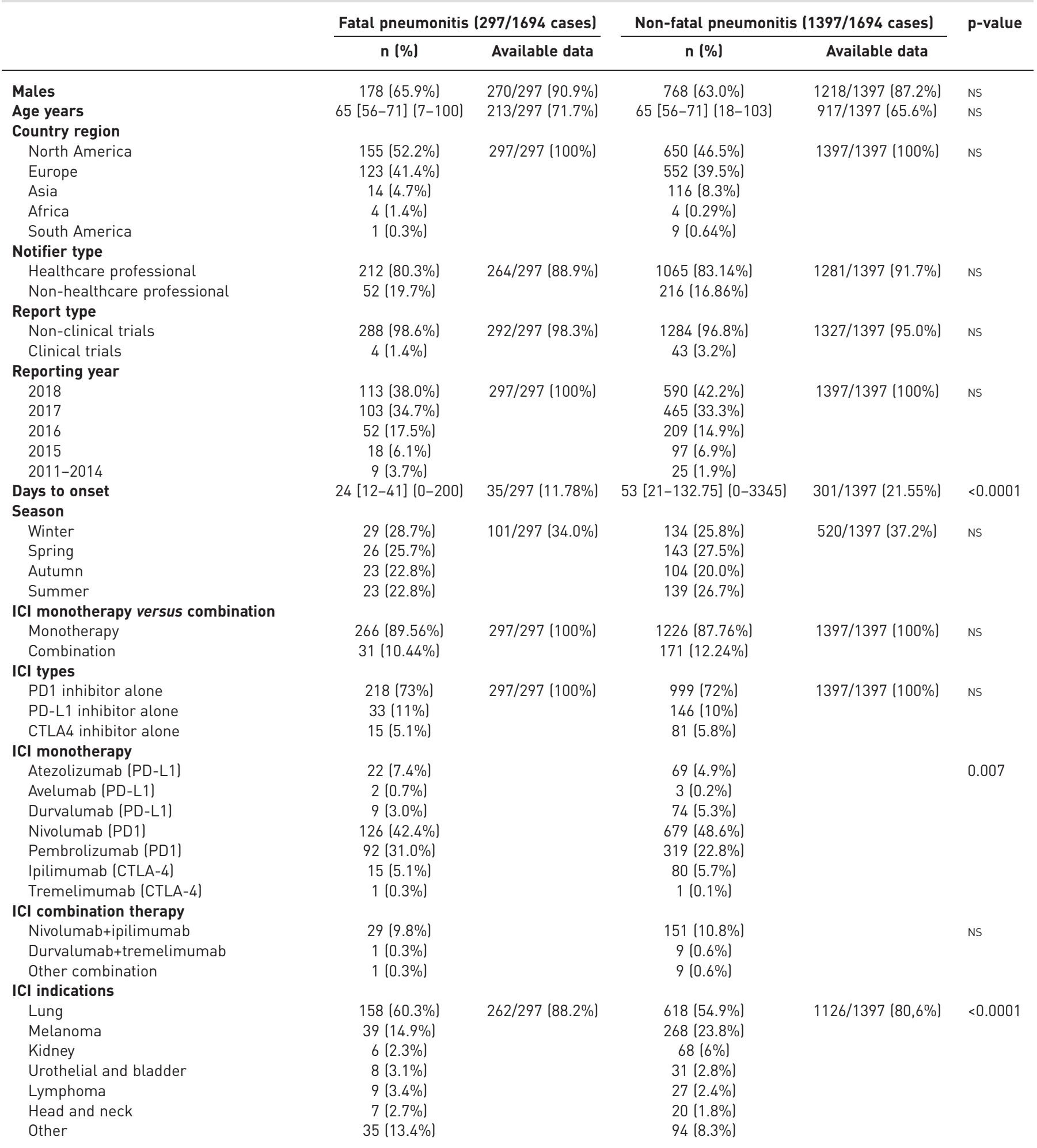




\begin{tabular}{|c|c|c|c|c|c|}
\hline & \multicolumn{2}{|c|}{ Fatal pneumonitis $(297 / 1694$ cases $)$} & \multicolumn{2}{|c|}{ Non-fatal pneumonitis (1397/1694 cases) } & p-value \\
\hline \multicolumn{6}{|l|}{ Concurrent conditions } \\
\hline Malignant progression & $66(22.2 \%)$ & & $97(6.9 \%)$ & & $<0.0001$ \\
\hline Infection/sepsis & $42(14.1 \%)$ & & $101(7.2 \%)$ & & $<0.0001$ \\
\hline Thromboembolism & $27(9.1 \%)$ & & $45(3.2 \%)$ & & $<0.0001$ \\
\hline Haemorrhage & $10(3.4 \%)$ & & $17(1.2 \%)$ & & 0.007 \\
\hline Renal injury & $10(3.4 \%)$ & & $17(1.2 \%)$ & & 0.007 \\
\hline \multicolumn{6}{|l|}{ Concurrent irAEs } \\
\hline Colitis & $24(8.1 \%)$ & $297 / 297(100 \%)$ & $90(6.4 \%)$ & $1397 / 1397(100 \%)$ & NS \\
\hline Haematotoxicity & $22(7.4 \%)$ & & $34(2.4 \%)$ & & $<0.0001$ \\
\hline Dermatitis & $20(6.7 \%)$ & & $85(6.1 \%)$ & & NS \\
\hline Diabetes & $6(2 \%)$ & & $15(1.1 \%)$ & & NS \\
\hline Myositis & $6(2 \%)$ & & $16(1.2 \%)$ & & NS \\
\hline Myocarditis & $5(1.7 \%)$ & & $4(0.3 \%)$ & & NS \\
\hline Stomatitis, sinusitis and nasopharyngitis & $5(1.7 \%)$ & & $11(0.8 \%)$ & & NS \\
\hline Nephritis & $3(1 \%)$ & & $16(1.2 \%)$ & & NS \\
\hline Peripheral neuropathy & $3(1 \%)$ & & $11(0.8 \%)$ & & NS \\
\hline Adrenalitis & $2(0.7 \%)$ & & $10(0.7 \%)$ & & NS \\
\hline Encephalitis and myelitis & $2(0.7 \%)$ & & $6(0.4 \%)$ & & NS \\
\hline Vasculitis & $2(0.7 \%)$ & & $3(0.2 \%)$ & & NS \\
\hline Cholangitis & $1(0.3 \%)$ & & $7(0.5 \%)$ & & NS \\
\hline Hypophysitis & $1(0.3 \%)$ & & $28(2 \%)$ & & NS \\
\hline Meningitis & $1(0.3 \%)$ & & $1(0.1 \%)$ & & NS \\
\hline
\end{tabular}

Data are presented as $\mathrm{n}(\%)$ or median [interquartile range] (range), unless otherwise stated. CTLA-4: cytotoxic T cell lymphocyte-associated protein-4; irAE: immune-related adverse events; PD-1/PD-L1: programmed cell-death protein-1/and its ligand.

not capture the number of individuals exposed to a given drug. Second, incomplete data in some cases precludes precise characterisation of demographics, clinical features and concomitant medical conditions that would be relevant to this patient population, such as radiation exposure or dosage, other lung disorders (COPD, interstitial or restrictive lung disease) or smoking history. In addition, database reporting is voluntary and thus, not all data fields are included in every report and quality might be variable.

In conclusion, our study highlights the increasing incidence of ICI- pneumonitis over the past few years with earlier TTO of fatal cases, emphasising the need for clinical vigilance of monitoring for ICI-pneumonitis events.

Melissa Y.Y. Moey $\oplus^{1}$, Paul Gougis $\odot^{2}$, Vincent Goldschmidt $\oplus^{2}$, Douglas B. Johnson ${ }^{3}$, Bénédicte Lebrun-Vignes ${ }^{2,3}$, Javid Moslehi $^{4}$, Jacques Cadranel ${ }^{5}$ and Joe-Elie Salem ${ }^{2,4}$

${ }^{1}$ Dept of Cardiovascular Sciences, Vidant Medical Center/East Carolina University, Greenville, NC, USA. ${ }^{2}$ Dept of Pharmacology, INSERM CIC-1901, UNICO-GRECO Cardio-oncology Program, CLIP2 Galilée, APHP.Sorbonne Université, Hôpital Pitié-Salpêtrière, Paris, France. ${ }^{3}$ Pitié and Saint Antoine Pharmacovigilance Centres, APHP, Sorbonne Université, UPEC EA EpiDermE 7379, Paris, France. ${ }^{4}$ Dept of Medicine, Vanderbilt University Medical Center, Nashville, TN, USA. ${ }^{5}$ Chest Dept, Thoracic Oncology Expert Center, Rare Pulmonary Diseases Constitutive Center, GRC \#4, Theranoscan Sorbonne Université and APHP, Sorbonne Université, Hôpital Tenon, Paris, France.

Correspondence: Jacques Cadranel, Service de Pneumologie, Hôpital Tenon, 4 rue de la Chine 75970, Paris, France. E-mail: jacques.cadranel@aphp.fr

Received: 11 Jan 2020 | Accepted after revision: 9 Feb 2020

Acknowledgements: The supplied data from VigiBase was obtained from a variety of sources. The likelihood of a causal relationship is not the same in all reports. The information does not represent the opinion of the Uppsala Monitoring Center or the World Health Organization. 
Conflict of interest: M.Y.Y. Moey has nothing to disclose. P. Gougis has nothing to disclose. V. Goldschmidt has nothing to disclose. D.B. Johnson reports grants from BMS and Incyte, and has been a member of advisory boards for BMS, Array Biopharma, Jansen, Novartis and Merck, outside the submitted work. B. Lebrun-Vignes has nothing to disclose. J. Moslehi reports personal fees for consultancy from BMS, Pfizer, Audentes and Nektar, during the conduct of the study; and has a patent for treating checkpoint inhibitor-induced adverse events pending. J. Cadranel reports grants and personal fees for advisory board work and participation in meetings from AZ, personal fees for advisory board work and participation in meetings from Roche, BMS and MSD, outside the submitted work. J-E. Salem reports personal fees from BMS, outside the submitted work; and has a patent for treating checkpoint inhibitor-induced adverse events pending.

\section{References}

1 Postow MA, Callahan MK, Wolchok JD. Immune checkpoint blockade in cancer therapy. J Clin Oncol 2015; 33: 1974-1982.

2 Wang DY, Salem JE, Cohen JV, et al. Fatal toxic effects associated with immune checkpoint inhibitors: a systematic review and meta-analysis. JAMA Oncol 2018; 4: 1721-1728.

3 Cadranel J, Canellas A, Matton L, et al. Pulmonary complications of immune checkpoint inhibitors in patients with non-small cell lung cancer. Eur Respir Rev 2019; 28: 190058.

4 Delaunay M, Cadranel J, Lusque A, et al. Immune-checkpoint inhibitors associated with interstitial lung disease in cancer patients. Eur Respir J 2017; 50: 1700050.

5 Salem JE, Manouchehri A, Moey M, et al. Cardiovascular toxicities associated with immune checkpoint inhibitors: an observational, retrospective, pharmacovigilance study. Lancet Oncol 2018; 19: 1579-1589.

6 Salem JE, Manouchehri A, Haugh AM, et al. Neurologic toxicity associated with immune checkpoint inhibitors: a pharmacovigilance study. J Immunother Cancer 2019; 7: 135.

7 Anquetil C, Salem JE, Lebrun-Vignes B, et al. Immune checkpoint inhibitor-associated myositis. Circulation 2018; 138: 743-745.

8 Rahouma M, Baudo M, Yahia M, et al. Pneumonitis as a complication of immune system targeting drugs? A meta-analysis of anti-PD/PD-L1 immunotherapy randomized clinical trials. J Thorac Dis 2019; 11: 521-534.

9 Karthik S, Psoter KJ, Voong KR, et al. Impact of checkpoint inhibitor pneumonitis on survival in NSCLC patients receiving immune checkpoint immunotherapy. J Thorac Oncol 2019; 14: 494-502.

10 Haratani K, Hayashi J, Chiba Y, et al. Association of immune-related adverse events with nivolumab efficacy in non-small-cell lung cancer. JAMA Oncol 2018; 4: 374-378.

$11 \mathrm{Su} \mathrm{Q}, \mathrm{Zhu} \mathrm{EC}, \mathrm{Wu} \mathrm{JB}$, et al. Risk of pneumonitis and pneumonia associated with immune checkpoint inhibitors for solid tumors: a systematic review and meta-analysis. Front Immunol 2019; 10: 10.

12 Nishino M, Giobbie-Hurder A, Hatabu H, et al. Incidence of programmed cell death 1 inhibitor-related pneumonitis patients with advanced cancer: a systematic review and meta-analysis. JAMA Oncol 2016; 2: $1607-1616$

13 McGovern K, Ghaly M, Esposito M, et al. Radiation recall pneumonitis in the setting of immunotherapy and radiation: a focused review. Future Sci OA 2019; 5: FSO378.

14 Sears CR, Pelkert T, Possick JD. Knowledge gaps and research priorities in immune checkpoint inhibitor-related pneumonitis: an official American Thoracic Society research statement. Am J Respir Crit Care Med 2019; 200: e31-e43.

15 Champiat S, Ferrara R, Massard C, et al. Hyperprogressive disease: recognizing a novel pattern to improve patient management. Nat Rev Clin Oncol 2018; 15: 748-762. 